\title{
A Bio-inspired Computer Fovea Model based on Hexagonal-type Cellular Neural Networks
}

\author{
C-H. Huang, Student Member, IEEE, H. Koeppl and C-T. Lin, Fellow, IEEE
}

\begin{abstract}
In this work we propose a novel computer fovea model based on hexagonal-type Cellular Neural Networks (hCNN). The hCNN represents a new image processing architecture that is motivated by the overwhelming evidence for hexagonal image processing in biological systems. The necessary new coupling templates and basic hCNN image operators are introduced. The fovea model includes the biological mechanisms of the photoreceptors, the horizontal cells, the ganglions, the bipolar cells, and their cooperation. Thus the model describes the signal processing from the optical stimulation at retina to the output of the ganglion cells. Different building blocks of the model turned out to be useful for practical image enhancement algorithms. Two such applications are considered in this work, namely the image sharpness improvement and the color constancy algorithm.
\end{abstract}

\section{INTRODUCTION}

The retina is a highly structured complex network of neurons. Hence, to analyze and to model its behavior is a highly challenging task. There has been a big research effort to understand the mechanisms in the retina in the last decades. In [1], [2] the information processing procedures in the retina both in space and time domain have been investigated. Based on this results a Gabor filtering simulator which is inspired by the biological behaviors of the retina has been proposed in [3]. The parallel processing structure of the retina and its implementation in terms of Cellular Neural Networks (CNN) is discussed in [4]. The investigations suggest that there are some kind of visual information enhancement algorithms present in the human vision system. Based on these previous results our work focuses on the modeling and simulation of the fovea based on hexagonal-type Cellular Neural Networks $(\mathrm{hCNN})$. The fovea is a small spot at the retina located approximately on the optical axis and is responsible for the acuity of the human vision.

There are five types of neurons distributed in five layers in retina [5]. The photoreceptors are in the outer nuclear layer, the horizontal cells, the amacrine cells and the bipolar cells are in the inner nuclear layer, and the ganglions cells are in the ganglion layer. The outer plexiform layer contains the synapse connections between the photoreceptors, the horizontal cells and the bipolar cells. The inner plexiform layer contains the synapse connections between the bipolar cells, the amacrine cells and the ganglion cells.

C-H. Huang is a Ph.D. Student at Department of Electrical and Control Engineering, National Chiao-Tung University, Taiwan (email:chhuang.ece90g@ nctu.edu.tw).

H. Koeppl is a Erwin Schrödinger postdoctoral fellow at the Dept. Electrical Engineering and Computer Sciences, University of California Berkeley, USA.

C-T. Lin is Professor and Dean, College of Computer Science Dean, Dept. Computer Science, National Chiao-Tung University, Taiwan.

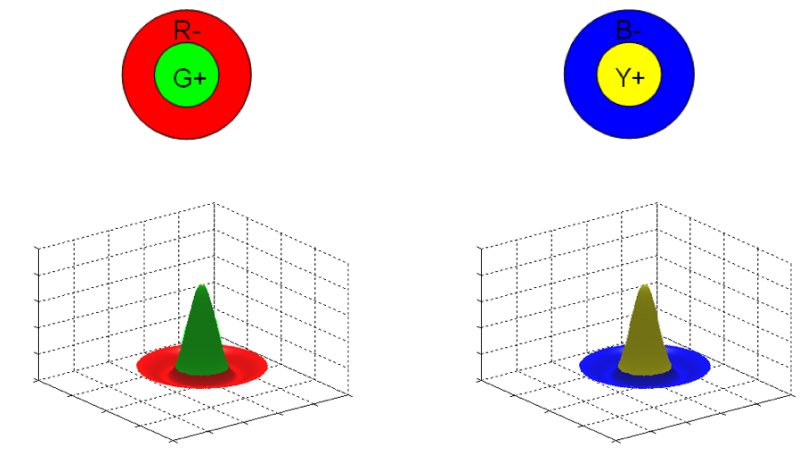

Fig. 1. Impulse responses of two different ganglion cells; for the centergreen-on/surround-red-off ganglion (left) and the center-yellow-on/surroundblue-off ganglion (right).

In the human vision system, photoreceptors can be classified into four types, namely the L-, M-, S-cone cells and the rod cells. Those kind of photoreceptors react to different wavelengths of the light. The rod cells can sense luminance information, and the L-, M-, and S-cone cell can detect the color information. It it is already known that those different kinds of photoreceptors co-work together and send the spikes to the ganglions via the bipolar cells and the horizontal cells [5]. Usually, one ganglion cell is activated by a set of photoreceptors consisting of more than one type. The differences in the set of photoreceptors result in a variation of the ganglion cells. The two major types of ganglion cells are called the center-on/surround-off and the center-off/surroundon ganglions.

In Fig. 1 the impulse response of a center-surround ganglion cell for two different colors is shown. The center of the group of the photoreceptors reacts to the stimulation in the different manner as the surround. Usually, that can be classified as red-green (RG) ganglions, blue-yellow (BY) ganglions, and black-white (BW) ganglions.

The early vision represents the set of first stage information processing mechanisms of the human vision system. Those mechanisms are operated in parallel across the visual field and are believed to be used for detecting the most basic visual features [5]. According to [6], the human vision system includes two fundamental features. The first one can be described in terms of a low-pass filtering operation across the visual field. In general, the result of the low-pass filtering represents the average intensity of a specific local area. This feature is referred to as the first order feature. The second feature is the difference between light intensities that project onto the retina. According to some investigations [5], [6], the 


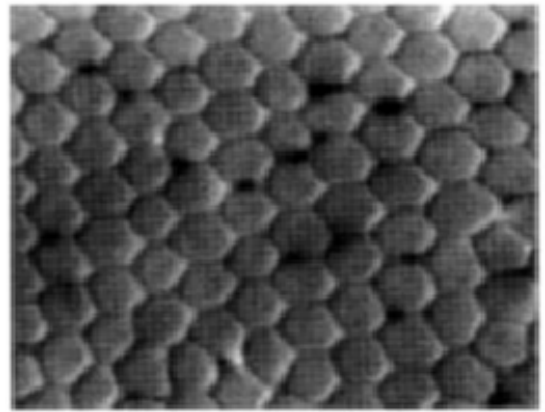

Fig. 2. Biological structure of the photoreceptor cells in the fovea of a mammalian, supporting the proposed hexagonal-type Cellular Neural Network.

boundary detection operation in the human vision system is based on this feature and is related to the zero crossing of the Laplacian of two Gaussians (LoG). Sometimes the result of this operation is called the second order feature.

The goal of this work is to model the Receptive Fields (RFs) of the cells in the fovea. From the point of view of the signal processing, a RF can be described as a finite impulse response spatial filter [1], [2]. Motivated by the biological evidence of hexagonal image processing structure in biological systems (confer to Fig. 2) and the efficient parallel processing architecture of the CNN we deploy the new hCNN to realize these spatial filters. In addition the work shows how image enhancement algorithms can be derived from the fovea model.

The work is organized as follows. Section II briefly reviews the CNN framework and introduces the novel bioinspired hexagonal-type $\mathrm{CNN}$ and proceeds with the definition of standard image operators for this architecture. The new computer fovea model and its submodels are discussed in section III. Simulation results and two image enhancement algorithms based in the fovea model are presented in section IV, while section $\mathrm{V}$ draws the conclusions.

\section{Cellular Neural Networks for Hexagonal IMAGE PROCESSING}

A CNN [7], [8] has already been proven to be a very powerful image processing kernel. It represents an alternative to a fully connected neural network and has evolved into a new paradigm for this type of array processors. The dynamic equation of a translation invariant planar $\mathrm{CNN}$ $\mathcal{C}: u(t, i, j) \mapsto y(t, i, j)$ is given by

$$
\begin{aligned}
\dot{x}(t, i, j) & =-x(t, i, j)+\sum_{k, l \in \mathcal{N}(i, j)} \bar{A}(i-k, j-l) y(t, k, l) \\
& +\sum_{k, l \in \mathcal{N}(i, j)} \bar{B}(i-k, j-l) u(t, k, l)+I,
\end{aligned}
$$

where

$$
y(t, i, j)=\frac{1}{2}(|x(t, i, j)+1|-|x(t, i, j)-1|) .
$$

\section{A. Hexagonal-type Cellular Neural Networks}

In (1) the Moore neighborhood for the coupling is assumed. A neighborhood of size one means that each cell is connected to its eight neighboring cells. Besides the change in the neighborhood size one can also change the lattice structure of the $\mathrm{CNN}$. One possible structure is a sixneighborhood structure such as the hexagonal-type structure [9]. A hexagonal-type Cellular Neural Network (hCNN) $\mathcal{H}$ : $u(t, \alpha) \mapsto y(t, \alpha)$ can be described by

$$
\begin{aligned}
\dot{x}(t, \alpha) & =-x(t, \alpha)+\sum_{\gamma \in \mathcal{N}(\alpha)} \hat{A}(\alpha-\gamma) y(t, \gamma) \\
& +\sum_{\gamma \in \mathcal{N}(\alpha)} \hat{B}(\alpha-\gamma) u(t, \gamma)+I,
\end{aligned}
$$

where $\alpha$ and $\gamma$ are now linear indices of the planar hexagonal lattice. The same output function (2) as for the rectangulartype structure is used. We introduce the following mathematical symbols to present the templates of the hCNN

$$
\begin{aligned}
& \hat{A}=\left\langle\begin{array}{ccc}
a_{3} & a_{2} \\
a_{4} & a_{0} & a_{1} \\
a_{5} & a_{6}
\end{array}\right\rangle \\
& \hat{B}=\left\langle\begin{array}{ccc}
b_{3} & b_{2} \\
b_{4} & b_{0} & b_{1} \\
b_{5} & b_{6}
\end{array}\right\rangle,
\end{aligned}
$$

where $\hat{A}$ and $\hat{B}$ is the feedback and control templates, respectively. Note that the indices in the templates are the same as in Middletons Hexagonal Image Processing (HIP) [10]. Subsequently, we will make use of the following notation. Assuming zero initial conditions a $\mathrm{CNN}$ or hCNN operator is defined by its feedback and control template as well as its bias. Thus, the definitions of specific operators $\operatorname{read} \mathcal{C}:\{\bar{A}, \bar{B}, I\}$ and $\mathcal{H}:\{\hat{A}, \hat{B}, I\}$, respectively.

\section{B. Stable Central Linear Systems and their Inverses}

If we consider the $\mathrm{CNN}$ as an image processor, then many linear properties can be analyzed. For example, the states located in the non-saturated region. In [11] a thorough analysis of the central linear $\mathrm{CNN}$ and its application to image processing is given. The same analysis can be done for the hCNN. Assume all the cells operate in the linear region. That is, $|x(t, \alpha)|<1$ for all $\alpha$, then the output equation (2) reduces to $y(t, \alpha)=x(t, \alpha)$. Thus, (3) can be simplified to

$$
\begin{aligned}
\dot{x}(t, \alpha) & =-x(t, \alpha)+\sum_{\gamma \in \mathcal{N}(\alpha)} \hat{A}(\alpha-\gamma) x(t, \gamma) \\
& +\sum_{\gamma \in \mathcal{N}(\alpha)} \hat{B}(\alpha-\gamma) u(t, \gamma)+I .
\end{aligned}
$$

Following [11] we absorb the first term of the right hand side of (6) in the feedback template by introducing the new linearized feedback and control templates $a$ and $b$, 
respectively as

$$
\begin{aligned}
& a=\hat{A}+\left\langle\begin{array}{ccc}
0 & 0 \\
0 & -1 & 0 \\
0 & 0
\end{array}\right\rangle \\
& b=\hat{B} .
\end{aligned}
$$

Denoting the spatial convolution operator on a planar hexag-

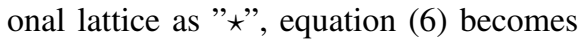

$$
\dot{x}(t, \alpha)=a(\alpha) \star x(t, \alpha)+b(\alpha) \star u(t, \alpha)+I .
$$

Applying the spatial discrete hexagonal Fourier transform [12] to (8) yields

$$
\dot{X}(t, \omega)=A(\omega) X(t, \omega)+B(\omega) U(t, \omega)+\delta(\omega) I,
$$

where capital letters represent the transforms of their lower letter counterparts. Appropriate zero-padding is applied where necessary. The variable $\omega$ represents the linear index of the hexagonal lattice in the transform domain. The set of coupled differential equation in (8) decouples through the application of the spatial transform. Thus for each fixed frequency location $\omega_{0}$, equation (9) is a linear first order differential equation for the time-evolution of $X\left(t, \omega_{0}\right)$. For symmetric templates $a$ and $b(9)$ is real valued for all $\omega$. Template symmetry will be assumed throughout the rest of this work. The stability condition is then obviously $A(\omega)<0$ for all $\omega$. For a constant input $u(\alpha) \equiv u(0, \alpha)=u(t, \alpha) \forall t$ the asymptotic state of a stable system is then given by

$$
X(\omega) \equiv \lim _{t \rightarrow \infty} X(t, \omega)=H(\omega) U(\omega),
$$

with

$$
H(\omega)=-\frac{B(\omega)}{A(\omega)}
$$

Thus, the asymptotic output $x(\alpha)$ is the result of the application of the spatial filter $H(\omega)$ to the input image $u(\alpha)$.

\section{CNN Image Operators}

A Laplace-like operation of a $\mathrm{CNN}$ is obtained by

$$
\bar{B}=\left[\begin{array}{ccc}
0 & -1 & 0 \\
-1 & 4 & -1 \\
0 & -1 & 0
\end{array}\right], \quad \bar{A}=\left[\begin{array}{lll}
0 & 0 & 0 \\
0 & 0 & 0 \\
0 & 0 & 0
\end{array}\right]
$$

and $I=0$. The zero-frequency component of the Fourier transform of the control template $\bar{B}$ is zero. This fact implies that the inversion of this operation will be quite difficult. Thus, we add a small positive constant $\epsilon^{2}$ to the central element and define the subsequently used Laplacelike operator $\mathcal{C}_{l \epsilon}$ as

$$
\mathcal{C}_{l \epsilon}:\left\{\left[\begin{array}{ccc}
0 & -1 & 0 \\
-1 & 4+\epsilon^{2} & -1 \\
0 & -1 & 0
\end{array}\right],\left[\begin{array}{lll}
0 & 0 & 0 \\
0 & 1 & 0 \\
0 & 0 & 0
\end{array}\right], 0\right\}
$$

For the HIP framework we introduce the corresponding Laplace-like operator as

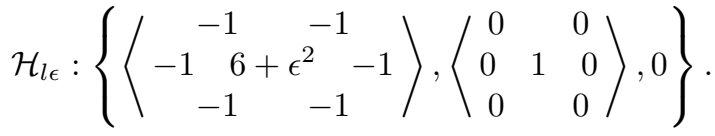

The inversion of $\mathcal{H}_{l \epsilon}$ (and correspondingly for $\mathcal{C}_{l \epsilon}$ ) can be obtained as

$$
H^{-1}(\omega)=-\frac{A(\omega)}{B(\omega)}=-\frac{-A(\omega)}{-B(\omega)},
$$

where the last equation should indicate that the linearized feedback template of the inverse operator $\mathcal{H}_{l \epsilon}^{-1}$ should be chosen to be $-B(\omega)$ to ensure the stability of the operator $\mathcal{H}_{l \epsilon}^{-1}$. The linearized control template of $\mathcal{H}_{l \epsilon}^{-1}$ should be chosen to be $-A(\omega)$. Thus, applying (7) the templates for $\mathcal{H}_{l \epsilon}^{-1}$ are

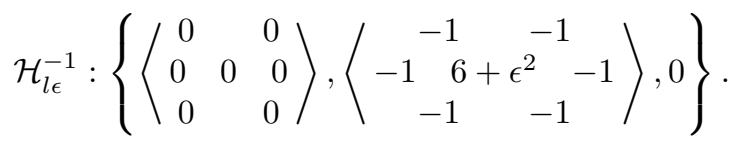

Note that operator $\mathcal{H}_{l \epsilon}^{-1}$ is the exact inverse of the operator $\mathcal{H}_{l \epsilon}$ defined in (14). The same inversion procedure can be applied to the rectangular operator $\mathcal{C}_{l \epsilon}$ of (13).

Another important image operator for a fovea model is the Gaussian operator. A Gaussian-like operation is performed by the rectangular $\mathrm{CNN}_{\mathcal{C}_{g \lambda}}[13]$ with

$$
\mathcal{C}_{g \lambda}:\left\{\left[\begin{array}{ccc}
0 & 1 & 0 \\
1 & -3-\lambda^{2} & 1 \\
0 & 1 & 0
\end{array}\right],\left[\begin{array}{ccc}
0 & 0 & 0 \\
0 & \lambda^{2} & 0 \\
0 & 0 & 0
\end{array}\right], 0\right\},
$$

where $\lambda^{2}$ is a small positive constant. The corresponding hexagonal operator $\mathcal{H}_{g \lambda}$ is defined by

$$
\mathcal{H}_{g \lambda}:\left\{\left\langle\begin{array}{ccc}
1 & 1 & \\
1 & -5-\lambda^{2} & 1 \\
1 & 1 & \rangle
\end{array}\right\rangle,\left\langle\begin{array}{ccc}
0 & 0 \\
0 & \lambda^{2} & 0 \\
0 & 0
\end{array}\right\rangle, 0\right\} .
$$

Following the above presented inversion procedure the inverses of the Gaussian-like operators $\mathcal{C}_{g \lambda}$ and $\mathcal{H}_{g \lambda}$ read

$$
\mathcal{C}_{g \lambda}^{-1}:\left\{\left[\begin{array}{ccc}
0 & 0 & 0 \\
0 & -\lambda^{2}+1 & 0 \\
0 & 0 & 0
\end{array}\right],\left[\begin{array}{ccc}
0 & 1 & 0 \\
1 & -4-\lambda^{2} & 1 \\
0 & 1 & 0
\end{array}\right], 0\right\}
$$

and

$$
\mathcal{H}_{g \lambda}^{-1}:\left\{\left\langle\begin{array}{cccc}
0 & 0 & \\
0 & \lambda^{2}+1 & 0 \\
0 & 0 &
\end{array}\right\rangle,\left\langle\begin{array}{ccc}
1 & 1 & \\
1 & -6-\lambda^{2} & 1 \\
1 & 1 &
\end{array}\right\rangle, 0\right\},
$$

respectively. Once again, note that the proposed operators (19) and (20) are the exact inverses of the operators in (17) and (18), respectively. This novel straightforward inversion of CNN image operators has relevance in many other application, e.g., the retinex algorithm [14] which can be modeled as a cascade of a high pass filter and threshold operator and the inverse of the first operator.

\section{The COMputer Fovea Model}

Since the retina is a highly structured network of the neurons, it became a quite valuable research topic in the fields of Human Computer Interaction. Many researchers provided summaries of the retinal structure and the biological evidences [15], [1], [2]. 


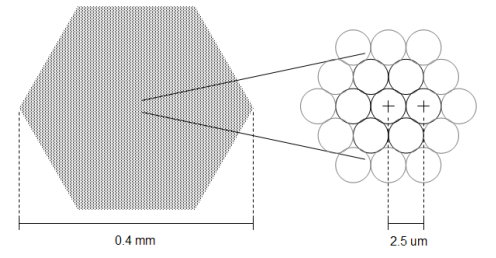

Fig. 3. Structure of the proposed computer fovea model.

The fovea locates on the center of the retina, and is the region of highest visual acuity. The fovea is a $0.2-$ $0.4 \mathrm{~mm}$ diameter rod-free area with very thin, densely packed photoreceptors. The photoreceptors in the fovea are nearly hexagonally arranged (see Fig. 2) [16], and the average of cone spacing (csp) has been estimated to about 2.5 to $2.8 \mu \mathrm{m}$. It is the most important area in the retina. The fovea is always directed towards whatever object one focuses on. In the fovea there are almost exclusively cones at their highest density. The structure of the proposed hCNN-based fovea model is shown in Fig. 3. The fovea model is constructed by a set of photoreceptors that are hexagonally arranged. In Fig. 4 the signal processing system of each cell in the fovea model is shown. In [3] is was suggested that because there are direct synaptic connections between the bipolar cells and the ganglion cells and only weak influence of the amacrin cells, the model of the bipolar cells can be incorporated into the model of the ganglion cells and the amacrin cells can be neglected. However, it is already known that the horizontal cells directly connect to the bipolar cells and have no direct connection to the ganglion cells [1], [2], [5]. Thus, we propose to keep the bipolar cells separated from the ganglion cells and consider the ganglion cell as a direct synaptic connection in our model.

\section{A. Photoreceptors}

For the sake of estimating the parameters of a fovea model, a simplified version of the proposed model in Fig. 4 is required and it is shown in Fig. 5. In the simplified version, we assume the differences between L-, M-, and S-cone cells are ignored, i.e., $x_{R}=x_{R}^{\prime}$. Meanwhile, since in this research, the ganglion is considered as a direct synaptic connection, we consider it as a all-pass filter.

The impulse response $g_{R}(i, j)$ of the photoreceptor can be represented as a Difference of two Gaussians (DoG). In the fovea, it can be described as a Gaussian function in the most cases [1], [2], [3], [5] with the standard deviation $\sigma$ of the Gaussian ranging from 1.5 to 12 (csp). In our approach, the CNN-based Gaussian-like operator of (17) is used to approximate the Gaussian filter

$$
\mathcal{C}_{g \lambda_{R}}(\delta(\alpha)) \approx g_{R}(\alpha),
$$

where $\lambda_{R}$ indicates the diffusion level of the Gaussianlike function. Now the question is how we set parameter $\lambda_{R}$ such that the final state of the CNN approximates the action of a Gaussian filter of predetermined $\sigma$. To obtain the corresponding values for $\lambda_{R}$ a Genetic Algorithm (GA) is

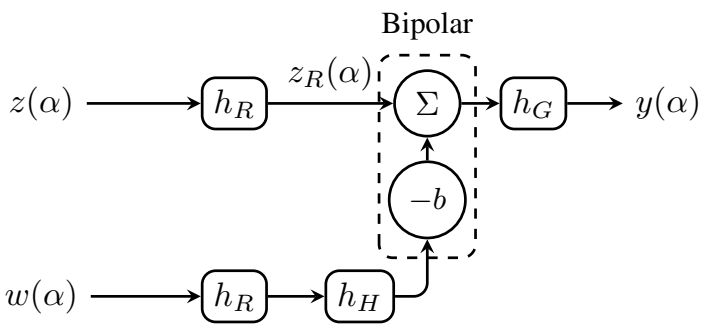

Fig. 4. The proposed fovea model including the photoreceptor cells, the horizontal cells, the bipolar cells and the ganglion cells.

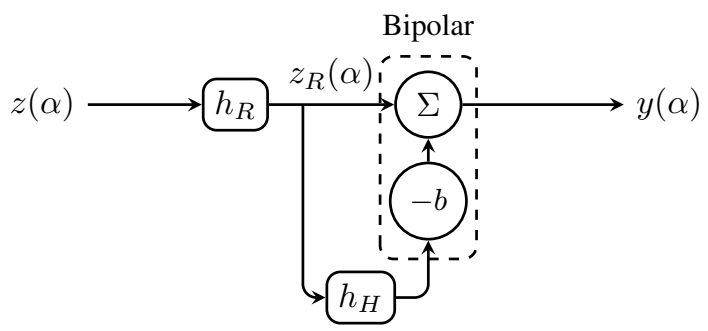

Fig. 5. The proposed simplified fovea model including the photoreceptor cells, the horizontal cells and the bipolar cells.

used. The correspondence is summarized in Tab. I. Clearly,

\begin{tabular}{|l|l|l|}
\hline$\sigma$ & $\lambda_{R}$ & MSE \\
\hline$\sqrt{2}$ & 0.5677 & $2.57 \times 10^{-5}$ \\
1.5 & 0.5360 & $2.85 \times 10^{-5}$ \\
12 & 0.0712 & $4.69 \times 10^{-9}$ \\
\hline
\end{tabular}

TABLE I

CORRESPONDING VALUE OF $\lambda_{R}$ FOR THE CNN-BASED APPROXIMATION OF A GAUSSIAN FILTER WITH STANDARD DEVIATION $\sigma$.

the RFs of the photoreceptor is used to determine the average intensity of a visual signal. In general, a photoreceptor acts like a low-pass filters. The outputs of the filters are the so called first order features.

\section{B. The Overall Fovea Response}

According to Fig. 5 the overall impulse response of the simplified fovea model reads

$$
h_{F}(\alpha)=h_{R}(\alpha) \star\left(\delta(\alpha)-b h_{H}(\alpha)\right) .
$$

Some physiological experiments indicated that the RF of the fovea system shows a center-surround characteristic. Even more, in [3] is it suggested that the RF of the overall fovea system can be modeled as the cascade of a Gaussian filter with $\sigma_{G}$ and a Laplace filter. According to [5], in the best lighting condition, the central part of RF is about $10 \mu \mathrm{m}(4$ csp). Similarly in [3] it is suggested that the standard deviation $\sigma_{G}$ of the Gaussian filter should be $\frac{2 c s p}{\sqrt{2}}=\sqrt{2}(\operatorname{csp})$. A combination of the CNN-based Laplace-like operator $\mathcal{H}_{l \lambda}$ and the $\mathrm{CNN}$-based Gaussian-like operator $\mathcal{H}_{g \epsilon}$ can be used to describe the overall system

$$
h_{F} \approx \mathcal{H}_{g \lambda}\left(\mathcal{H}_{l \epsilon}(\delta(\alpha))\right)
$$




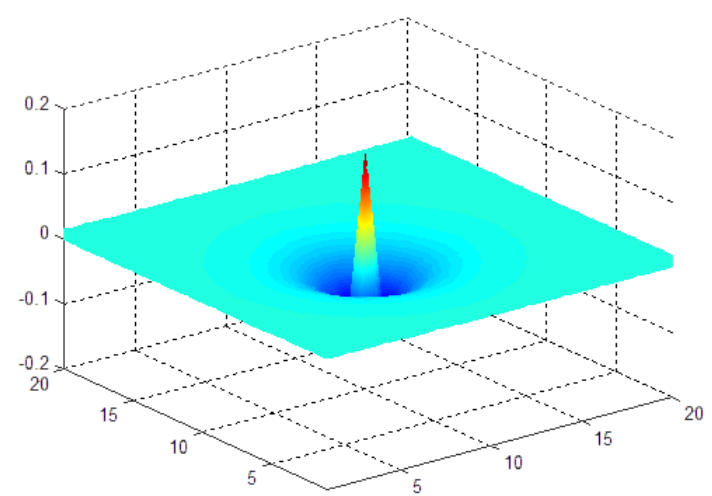

Fig. 6. Radial symmetric impulse response of the hCNN model of the horizontal cell with $\lambda_{R}=0.536, \lambda_{G}=0.568, \epsilon=0.01$ and $b=0.75$.

where $\epsilon$ is a small nonzero value and $\lambda$ is obtained via the GA.

\section{The Horizontal Cells}

Using (22) the impulse response of a horizontal cell $h_{H}(\alpha)$ is given by

$$
h_{H}(\alpha)=\frac{1}{b}\left(\delta(\alpha)-h_{R}^{-1}(\alpha) \star h_{F}(\alpha)\right) .
$$

Applying the Gaussian model for the photoreceptor $h_{R}(\alpha)$ and (23) for the overall fovea model, the horizontal cells can be estimated by (24). In terms of hCNN operator approximation one obtains $h_{H}(\alpha) \approx \mathcal{H}_{H}(\delta(\alpha))$ with

$$
\mathcal{H}_{H} \equiv \frac{1}{b}\left(1-\mathcal{H}_{g \epsilon}^{-1} \circ \mathcal{H}_{g \lambda} \circ \mathcal{H}_{l \epsilon}\right),
$$

where "o" denotes the composition of operators. Having identified each impulse response of the simplified fovea model, one can apply the filters to the two-input channel model of Fig. 3. In this case the response of the overall fovea model in terms of hCNN operators reads

$$
y(\alpha) \approx \mathcal{H}_{g \lambda}(z(\alpha))-b \mathcal{H}_{g \lambda}\left(\mathcal{H}_{H}(w(\alpha))\right),
$$

which represents the final model equation.

\section{Simulation Results}

\section{A. The Biology-related Response and the Illumination}

In Fig. 6 an impulse response of the horizontal cell in the hCNN-based fovea model is shown. This can be compared with the well known RF structure in Fig. 1. One specific simulation result is shown in Fig. 7 where the input image is combination of a black and a white strip. The graph represents the intensity along the cross-section of the two stripes. The behavior of this model is quite similar to that of the human vision system (see Fig. 1). Another example of the characteristics of the proposed hCNN fovea model for a natural image are shown in Fig. 8, Fig. 9, Fig. 10 and Fig. 11. In order to present the effect of the hexagonal lattice, the resultion of these images is reduced. In accordance to Fig. 5

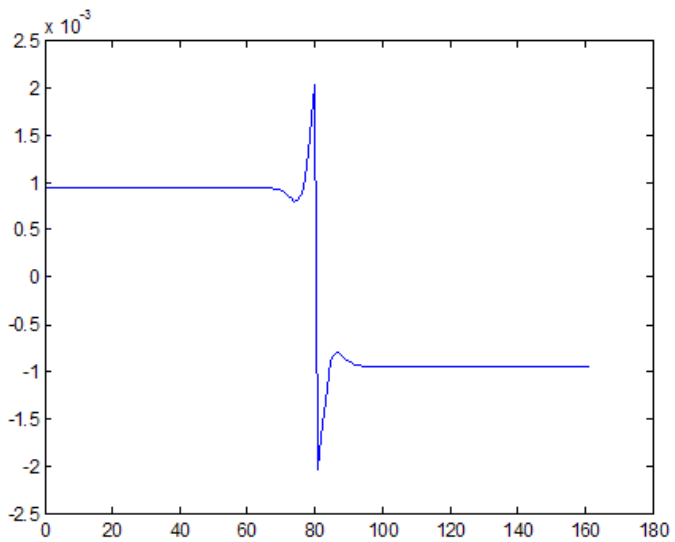

$\alpha$

Fig. 7. Cross-section of the output image of the hCNN fovea model for the input image containing a black and a white stripe; note that the beavers of this model are quite similar to that in the human vision system (see Fig. 1).

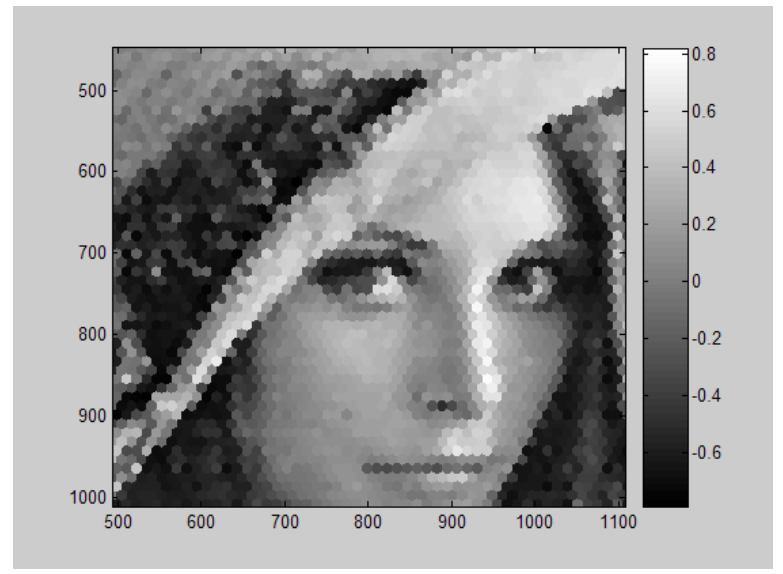

Fig. 8. Hexagonal input image to the proposed hCNN fovea model.

the input image Fig. 8 is passed through the photoreceptor cells and the horizontal cells and results in the Fig. 9. The overall response of the fovea (subtraction of a scaled version of the above image from the output of the photoreceptor) is shown in Fig. 10. Because the photoreceptor is just a Gaussian filter (see (21)) its output it not shown here. In addition the output of the rectification is given in Fig. 11.

\section{B. Image Sharpness Improvement Algorithm}

In the human vision system the second order feature provides the related difference information between cells. That is why the human vision system can identify the same texture in different light conditions. Following this, in [17] a sharpness improvement algorithm based on adaptive edge detection is proposed using multiple Gaussian derivative filters. Based on their idea, we can simply use the responses of the hCNN fovea model to implement sharpness improvement. For the luminance channel of CIE-L*a*b* the operation of the impulse response

$$
h_{s}(\alpha)=\delta(\alpha)+\gamma_{1} h_{F, \epsilon, \lambda_{1}}(\alpha)+\gamma_{n} h_{F, \epsilon, \lambda_{n}}(\alpha), L(\alpha)=\text {, }
$$




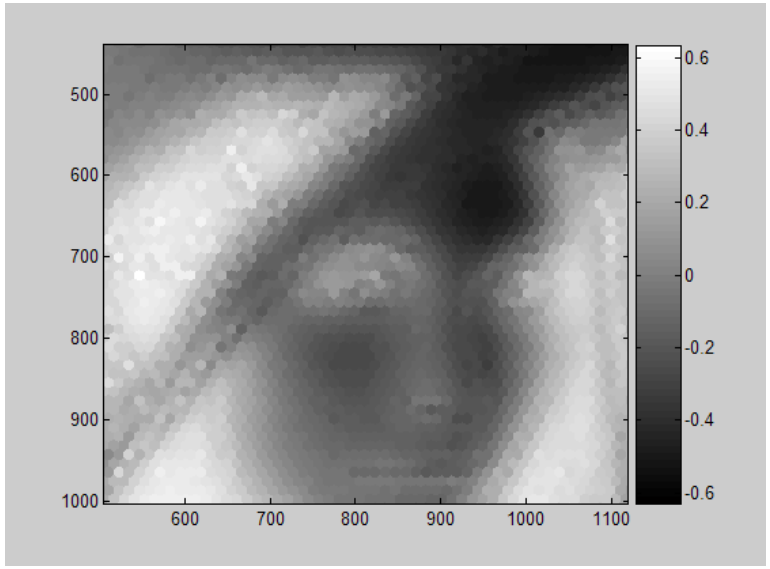

Fig. 9. Output of the cascade of the photoreceptor and the horizontal cells in response to the input given in Fig. 8.

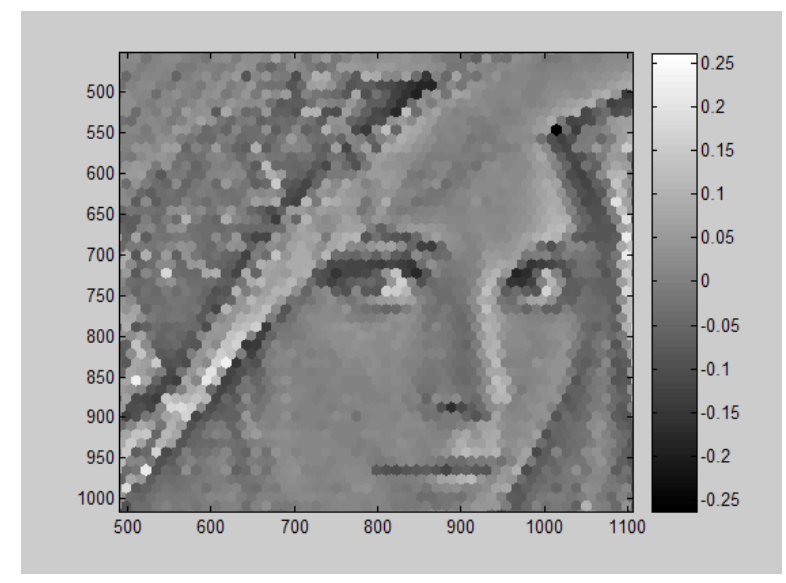

Fig. 10. Output of the complete hCNN fovea model in accordance to the block diagram Fig. 4 in response to the input image given in Fig. 8.

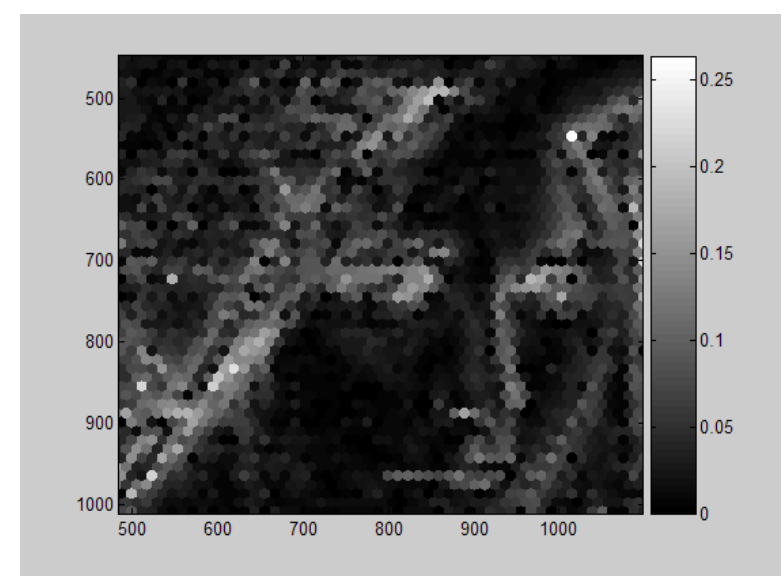

Fig. 11. Output of the complete hCNN fovea model followed by the rectification procedure in response to the input image given in Fig. 8.

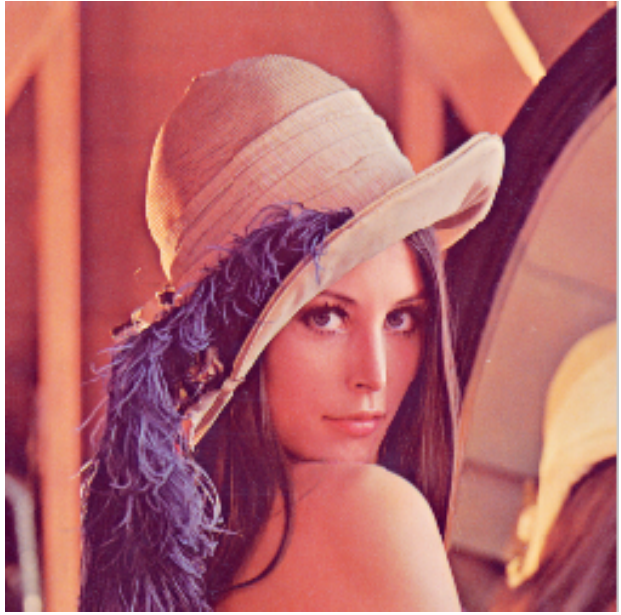

Fig. 12. Input image for the hCNN fovea model based sharpness improvement algorithm.

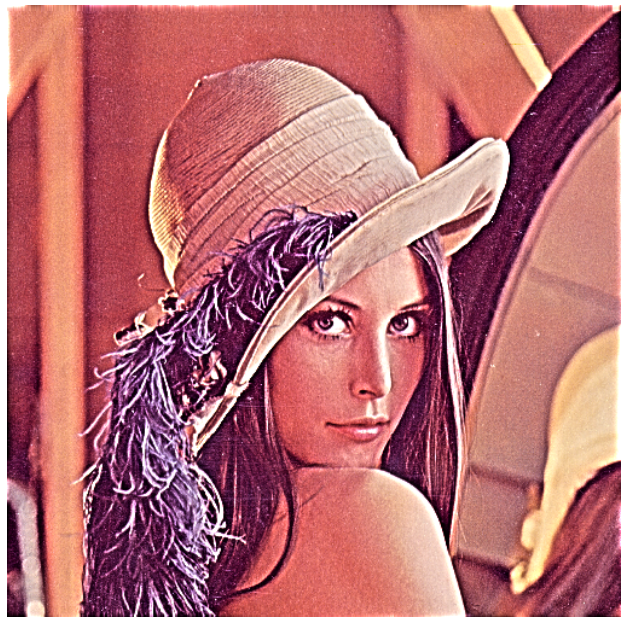

Fig. 13. Result of the hCNN fovea model based sharpness improvement algorithm with the input image given in Fig. 12.

yields a sharpness improved image, where $\gamma_{1}, \ldots, \gamma_{n}$ represent the adaptive weights and $\lambda_{1}, \ldots, \lambda_{n}$ are the different scale parameters of the different fovea channels given by their impulse responses $h_{F, \epsilon, \lambda_{n}}$. In Fig. 12 and Fig. 13 the input and the output of sharpness improvement algorithm for an sample image are shown, respectively.

\section{Color Constancy Algorithm}

According to the gray world hypothesis, the average color of the world can be considered as gray [18], [19]. Based on this assumption, the features of the photoreceptor can be used to estimate the light shifting. Even more, we are able to remove the light shifting by this model. The corrected color channels $\hat{a}_{1}(\alpha)$ and $\hat{b}(\alpha)$ of the inputs $\bar{a}(\alpha)$ and $\bar{b}(\alpha)$, respectively compute to

$$
\begin{aligned}
& \hat{a}(\alpha)=\xi_{a}\left(\bar{a}(\alpha)-\mathcal{H}_{g \lambda_{R}}\left(\bar{a}_{1}(\alpha)\right)+\beta_{a}\right. \\
& \hat{b}(\alpha)=\xi_{b}\left(\bar{b}(\alpha)-\mathcal{H}_{g \lambda_{R}}\left(\bar{b}_{1}(\alpha)\right)+\beta_{b},\right.
\end{aligned}
$$



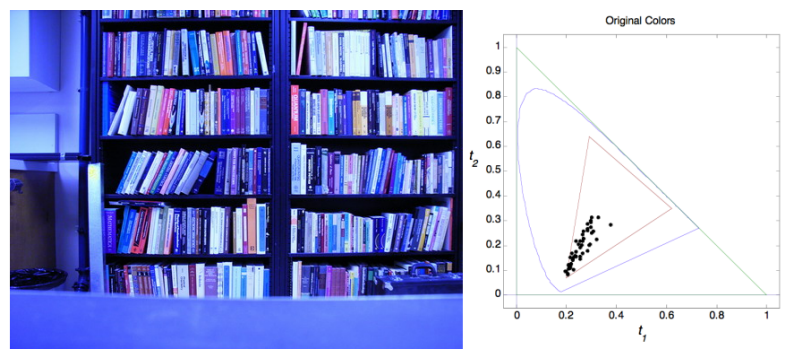

Fig. 14. Input image with a color shift (left) indicated in the color chromatic diagram (right)
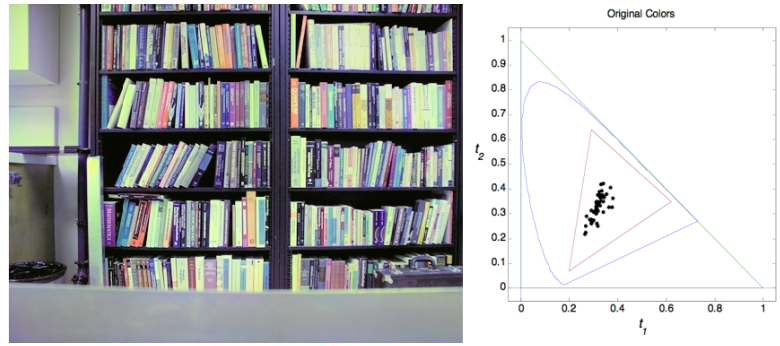

Fig. 15. Corrected version of the image in Fig. 14 applying the hCNN-based color constancy algorithm of (28) (left) and its color chromatic diagram (right).

where $\left(\xi_{a}, \xi_{b}\right)$ are scales parameters and $\left(\beta_{a}, \beta_{b}\right)$ corresponds to the definition of gray. In some environments the color shift of the input image can be quite extreme and the ranges of the color space are restrained. In those cases the parameters $\xi_{k}$ are required in order to restore the image. In Fig. 14 and Fig. 15 shows a color shifted input and its corrected version applying the hCNN-based color constancy algorithm, respectively.

\section{CONCLUSions}

In this paper we investigate the implementation of a fovea model based in a hexagonal-type Cellular Neural Network. Although, not all biological details of the mammalian fovea are known at present we are able to roughly model the behavior of the fovea and consequently find possible applications of the model for image enhancement algorithms. The proposed overall fovea model includes models for the photoreceptor cells, the horizontal cells and the bipolar cells. Simulation result confirm the validity of the discussed model. Image enhancement algorithms are deduced from the fovea model, such as the sharpness improvement algorithm as well as the color constancy algorithm.
The derivation of possible image processing applications from biological mechansims in the retina is one of the interests of our future research.

\section{REFERENCES}

[1] S. Shah and M. D. Levine, "Visual information processing in primate cone pathways - part I: a model," IEEE Transactions on Systems, Man, and Cybernetics, vol. 26, no. 2, pp. 259-274, 1996.

[2] —, "Visual information processing in primate cone pathways -part II: experiments," IEEE Transactions on System, Man, and Cybernetics - Part B: Cybernetics, vol. 26, no. 2, pp. 275-289, 1996.

[3] J. Thiem and G. Hartmann, "Biology-inspired design of digital Gabor filters upon a hexagonal sampling scheme," in International Conference on Pattern Recognition, Barcelona, Spain, 2000, pp. 445-448.

[4] D. Balya, B. Roska, T. Roska, and F. S. Werblin, "A CNN framework for modeling parallel processing in a mammalian retina," International Journal on Circuit Theory and Applications, vol. 30, pp. 363-393, 2002.

[5] D. Hubel, Eye, brain, and vision. New York: Scientific American Library, 1988.

[6] A. K. Jain and F. Farrokhnia, "Unsupervised texture segmentation using Gabor filters," Pattern Recognition, vol. 24, no. 12, pp. 11671186, 1991.

[7] L. O. Chua and L. Yang, "Cellular neural networks: theory," IEEE Transactions on Circuits and Systems, vol. 35, no. 10, pp. 1257-1272, 2005.

[8] — "Cellular neural networks: applications," IEEE Transactions on Circuits and Systems, vol. 35, no. 10, pp. 1273-1290, 1988.

[9] C. H. Huang and C. T. Lin, "Cellular neural networks for hexagonal image processing," IEEE Transactions on Circuits and Systems - I: Special Issue on Advances on Life Science Systems, submitted, 2005.

[10] L. Middleton and J. Sivaswamy, "A framework for practical hexagonalimage processing," Journal on Electronic Imaging, vol. 11, no. 1, pp. 104-114, 2002.

[11] K. R. Crounse and L. O. Chua, "Methods for image processing and pattern formation in cellular neural networks: a tutorial," IEEE Transactions on Circuits and Systems - I, vol. 42, no. 10, pp. 583601, 1995.

[12] L. Middleton, J. Sivaswamy, and G. Coghill, "The fft in a hexagonalimage processing framework," in New Zealand Conference on Image and Vision Computing, Dunedin, 2001, pp. 231-236.

[13] H. Kobayashi, J. L. White, and A. A. Abidi, "An active resistor network for gaussian filtering of images," IEEE Journals on SolidState Circuits, vol. 26, pp. 738-748, 1991.

[14] B. K. P. Horn, "Determining lightness from an image," Computer Graphics and Image Processing, vol. 3, no. 1, pp. 277-299, 1974.

[15] R. W. Rodieck, "The primate retina," Comparative Primate Biology, Neurosciences, vol. 4, pp. 203-278, 1988

[16] J. I. Yellot, "Spectral consequences of photoreceptor sampling in the rhesus retina," Science, vol. 212, pp. 382-385, 1983.

[17] H. Kotera, Y. Yamada, and K. Shimo, "Sharpness improvement adaptive to edge strength of color image," in Eighth Color Imaging Conference: Color Science and Engineering Systems, Technologies, Applications, 2000, pp. 149-154.

[18] R. Gershon, A. D. Jepson, and T. K. Tsotsos, "From [R,G,B] to surface reflectance: computing color constant descriptors in images," in Proceeding of the International Conference on Artificial Intelligence, vol. 2, 1987, pp. 755-758.

[19] G. Buchsbum, "A spatial processor model for object colour perception," Journal of the Franklin Institute, vol. 310, pp. 337-350, 1980. 